\title{
Directed Search of Biologically Active Compounds among Hydrogenated Isoindolylalkyl(alkylaryl-,aryl-)carboxylic Acids with Quinazoline Fragment that Modify the Carbohydrate Metabolism: Design, Synthesis and Modification
}

\author{
Yulya Victorivna Martynenko, ${ }^{1}$ Oleksii Mykolayovich Antypenko, ${ }^{1}$ \\ Oleksandr Anatolievich Brazhko, ${ }^{2}$ Irina Borisivna Labenska ${ }^{2}$ \\ and Sergii Ivanovich Kovalenko ${ }^{1, *}$ \\ ${ }^{1}$ Organic and Bioorganic Chemistry Department, Zaporizhzhya State Medical University, 26, Mayakovsky Ave., \\ Zaporizhzhya, 69035, Ukraine \\ ${ }^{2}$ Chemistry Department, Zaporizhzhia National University, 62, Gogol st., Zaporizhzhia, 69095, Ukraine \\ *Corresponding author: E-mail: kovalenkosergiy@gmail.com
}

Received: 10-01-2018

\begin{abstract}
An effective synthesis of (3H-quinazoline-4-ylidene)hydrazides of $N$-carboxyalkyl-(arylalkyl-,aryl-)isoindoline-1,3-diones, using activated $N$-protected aminoacids and 4-hydrazinoquinazoline was proposed in the framework methodology of directed search of hypoglycemic agents (fragment-oriented design, molecular docking). These hydrazides prepared via cyclocondensation under acid catalysis were converted to the corresponding 2-([1,2,4]triazolo[1,5-c]quinazoline-2-yl-) alkyl-(alkylaryl-,aryl-)-hydroisoindole-1,3(2H)-diones. The structure of synthesized compounds was established using IR, ${ }^{1} \mathrm{H}$ and ${ }^{13} \mathrm{C}$ NMR spectroscopy and LC-MS and the features of spectral pattern were discussed. The results of pharmacological screening revealed a series of compounds, that are short-acting hypoglycemic agents like prandial regulators of glucose (Mitiglinide). The SAR analysis showed, that the introduction of a hydrogenated 1,3-dioxoisoindole moiety bonded through linker groups with 4-hydrazinoquinazoline and triazolo[1,5-c]quinazoline cycles is reasonable in the context of searching for short-acting hypoglycemic agents and requires further research.
\end{abstract}

Keywords: (3H-quinazoline-4-ylidene)hydrazides of $N$-carboxyalkyl-(arylalkyl-,aryl-)isoindoline-1,3-diones; 2-([1,2,4] triazolo[1,5-c] quinazoline-2-yl-)alkyl-(alkylaryl-,aryl-)hydroisoindole-1,3(2H)-diones; directed search; physico-chemical properties; spectral features; hypoglycemic activity

\section{Introduction}

Quinazoline derivatives and their condensed analogues have always attracted the attention of medicinal chemists as objects of advanced research, aimed at the elaboration of new drugs. This fact is explained by the high biological activity of natural, ${ }^{1-3}$ as well as synthetic quinazolines $^{4-19}$ and, undoubtedly, their wide ability to chemical modification. ${ }^{10-13,18}$ In recent years, the interest for this heterocyclic system has increased greatly since the introduction of in silico approaches to the drug search strategy, combinatorial chemistry and high-throughput screening.
These approaches have led to the discovery and creation of a number of lead-compounds and original drugs with antitumor, antihypertensive, diuretic, hypoglycemic and other biological activities.,6,7,17 It should be noted, that information about the biological targets and fragment-oriented design based on them ${ }^{20,21}$ has played a key role in the creation of therapeutic molecule data. Using the above-mentioned search strategy we performed an attempt to one of the fragments-oriented design approaches based on the available results of hypoglycemic activity of $\mathrm{N}$-carboxyalkyl-(arylalkyl-,aryl-) substituted hydrogenated isoindoline-1,3-diones, ${ }^{22}$ known hypoglycemic agents 
(Mitiglinide 23,24 $^{\text {and Apabetalone }} \mathrm{e}^{25}$ ) and virtual screening. To enhance the pharmacological activity a combination in the same molecule of these compounds with a suitable pharmacophore quinazoline fragment was used (Figure 1).

Thus, the aim of this work is a directed search for biologically active compounds, that modify the carbohydrate metabolism, based on unknown ( $3 \mathrm{H}$-quinazoline-4-ylidene)hydrazides of hydrogenated isoindolylalkyl(alkylaryl-,aryl-)carboxylic acids, their further modification toward triazoloquinazolines, the study of their structural peculiarities and establishment of the structure-activity relationship (SAR) for further optimization of their structure.

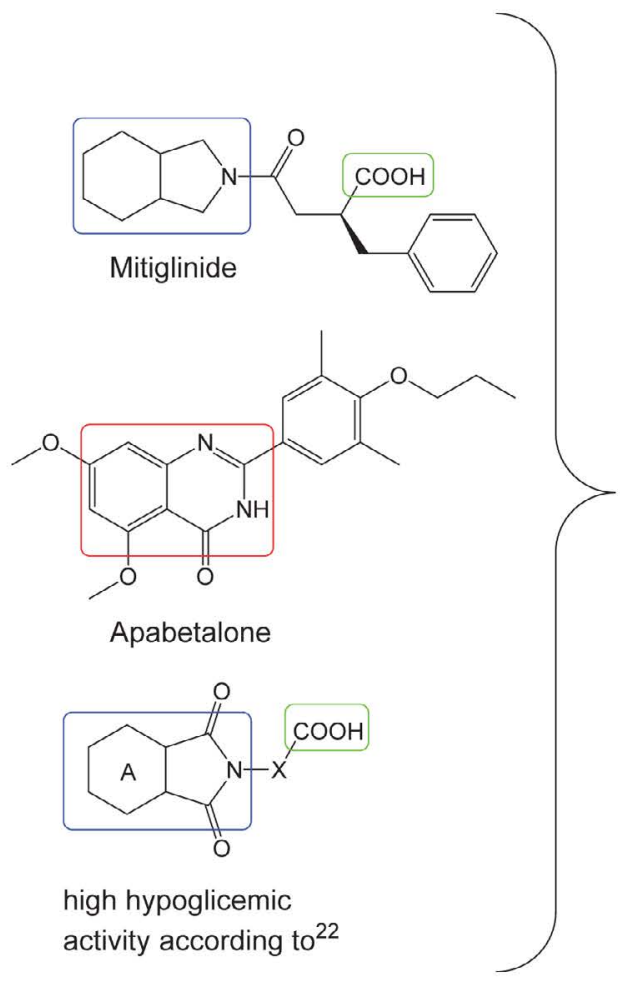

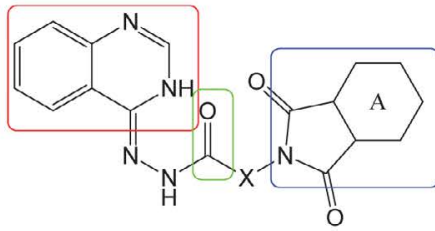

pharmacophore combination

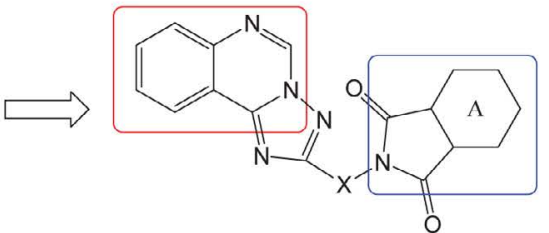

derivatization ical ionization - APCI). Ionization mode was a concurrent scanning of positive and negative ions in the mass range $80-1000 \mathrm{~m} / \mathrm{z}$.

Substances 1a-i were synthesized according to the reported procedures. ${ }^{22}$ Other starting materials and solvents were obtained from commercially available sources and used without additional purification.

\section{2. Molecular Docking}

Pancreatic beta-cell KATP channel (PDB ID - 6BAA) was used as the target protein to carry out molecular docking. Metglitinide and Glibenclamide were used as the reference drugs. The chemical formula of Glibenclamide was obtained from the mentioned PDB-file for calculating affinity value, and structure of Metglitinide was prepared as others ligands. ${ }^{26}$

Ligand preparation. Substances were constructed using Marvin Sketch 17.21 and saved as mol. files. Subsequently, using the Marvin Sketch 17.21 program, ${ }^{27}$ they
Figure 1. The search strategy for hypoglycemic agents based on the fragment-oriented design (combination of isoindole and quinazoline moieties in molecule).

\section{Experimental Section}

\section{1. Chemistry \\ General Methods}

Melting points were determined in open capillary tubes in a Stuart SMP30 apparatus and are uncorrected. The elemental analyses $(\mathrm{C}, \mathrm{H}, \mathrm{N})$ were performed using the ELEMENTAR vario EL cube analyzer. IR spectra (4000-600 $\mathrm{cm}^{-1}$ ) were recorded on a Bruker ALPHA FTIR spectrometer using a module ATR eco ZnSe. ${ }^{1} \mathrm{H}$ NMR spectra $(400 \mathrm{MHz})$ were recorded on Varian-Mercury 400 spectrometer with $\mathrm{SiMe}_{4}$ as the internal standard in DMSO- $d_{6}$ solution. LC-MS spectra were recorded using chromatography/mass spectrometric system, which consists of a high-performance liquid chromatograph Agilent 1100 Series equipped with diode-matrix and a mass-selective were optimized with the help of the molecular-mechanical $\mathrm{MM}+$ algorithm in conjunction with the semiempirical PM3 method of molecular modeling with the maximum number of cycles and the Polak-Ribiere algorithm. Molecular mechanics is used to obtain more realistic geometric values for most organic molecules, since it has a large number of parameters. The next step was to re-optimize MM+-optimized structures with using semiempirical PM3 molecular modeling method and the preservation of molecules in PDB files. Using Auto Dock Tools-1.5.6 these PDB files were converted to PDBQT while the rotational number of link options was typical.

Protein preparation. PDB files have been downloaded from a data bank of proteins. ${ }^{26}$ Discovery Studio v17.2.0.16349 was used to remove water molecules and ligands from the file. After that, the protein was saved as PDB 
file. Polar hydrogen atoms were added and the protein was saved as PDBQT into Auto Dock Tools-1.5.6. The search grid for docking the protein was set as following: center_x $=113$, center_y $=191$, center_z $=128$, size_x $=22$, size_y $=22$, size $\_z=22$. Vina was used for proper docking. Discovery Studio v17.2.0.16349 was used for visualization. ${ }^{28}$

\section{3. Pharmacology}

\section{3. 1. Hypoglycemia Activity Test}

Study of hypoglycemic activity was conducted on 150 Wistar white rats (male, weight $100-180$ g, age 3.5 month) which were obtained from nursery of PE Biomodelservice (Kiev, Ukraine). Experiments on animals were done according to the bioethics principles. ${ }^{23}$ Selected after quarantine animals by random sampling were divided in groups of 6 male rats on the assumption of absence of external signs of diseases and homogeneity by weight $( \pm 15 \%)$. Experimental animals were not fed during 12 hours before injection of studied compounds. The weight of all animals was measured before experiment. Intragastric injection of studied compounds was conducted using atraumatic probe as water solution or finely dispersed suspension stabilized by Tween- 80 in a dose of $10 \mathrm{mg} / \mathrm{kg}$. Intact and control groups received equivalent volume of water by the same way. Hypoglycemic activity of the synthesized compounds was evaluated via changes of glucose level before and after injection of the studied substances. Measurements of glucose level were carried out 2, 4 and 6 hours after injection. Metformine $(50 \mathrm{mg} / \mathrm{kg}$ ) was used as a reference drug.

Before the experiment the compounds were injected as an aqueous solution or 3-5\% micronized water suspension, stabilized by Tween- 80 in a dose of $10 \mathrm{mg} / \mathrm{kg}$ using atraumatic probe in order to identify prandial hypoglycemic agents. Subsequently, after 60 minutes, $40 \%$ glucose solution was injected to rats at a dose of $3000 \mathrm{mg} / \mathrm{kg}$. Hypoglycemic activity of the synthesized compounds was evaluated via changes of glucose level before and after injection of substances. Measurements of glucose level were carried out in 15, 30, 60 and 120 minutes after injection.

Statistical analysis was done using standard software complex, namely Microsoft Office Excel 2003 and STATISTICA ${ }^{\circledast}$ for Windows 6.0 (StatSoft Inc., № AXXR712D833214 FAN5). For each estimated value arithmetic mean $(\mathrm{M})$, and standard error of the mean $( \pm \mathrm{m})$ were defined. During verification of statistical hypothesis, null hypothesis were declined if statistical criterion $p<0.05$.

General Procedure for the Synthesis of ( $3 \mathrm{H}$-Quinazoline -4-ylidene)hydrazides $N$ - Carboxyalkyl-(arylalkyl-, aryl-)isoindoline-1,3-diones (3a-i)

$1.62 \mathrm{~g}(0.01 \mathrm{~mol})$ of $N, N^{\prime}$-carbonyldiimidazole was added to a suspension of $0.01 \mathrm{~mol}$ of the corresponding 2-(isoindoline-1,3-dione)alkyl-(alkyl-,arylalkyl-,aryl-) carboxylic acids $(\mathbf{1} \mathbf{a}-\mathbf{i})$ in $30 \mathrm{~mL}$ of dioxane. The mixture was kept at temperature $60-70{ }^{\circ} \mathrm{C}$ for $50-60$ minutes until the carbon dioxide was completely released. After addition

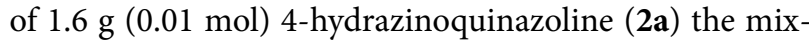
ture was kept at a room temperature overnight or at 80 ${ }^{\circ} \mathrm{C}$ for 1.5 hour. Dioxane was distilled off. The water was added and the mixture was neutralized by $0.1 \mathrm{M}$ aqueuos hydrochloric acid to $\mathrm{pH}$ 6-7. The solid product was filtered in the case of residue formation. If it was necessary, compounds were crystallized.

2-(1,3-Dioxooctahydro-2H-isoindole-2-yl)-N'-(quinazoline-4(3H)-ylidene)propanehydrazide (3a). Yield 61.9\%; m.p. $100-102{ }^{\circ} \mathrm{C}$; IR $\left(\mathrm{cm}^{-1}\right)$ : $3297,2917,2849,2693,2353$, $1704,1615,1550,1472,1386,1194,1130,984,759,689 ;{ }^{1} \mathrm{H}$ NMR $\delta$ (ppm): $11.46(\mathrm{~s}, 1 \mathrm{H}, 3-\mathrm{NH}), 10.16 / 10.03(\mathrm{~s}, 1 \mathrm{H}$, NHCO), 7.80-7.61 (m, 2H, H-2,5), $7.37(\mathrm{t}, J=7.9 \mathrm{~Hz}, 1 \mathrm{H}$, $\mathrm{H}-7$ ), 7.19 (t, $J=7.9 \mathrm{~Hz}, 1 \mathrm{H}, \mathrm{H}-6), 7.10(\mathrm{~d}, J=8.0 \mathrm{~Hz}$, $1 \mathrm{H}, \mathrm{H}-8), 5.15 / 4.75\left(\mathrm{q}, J=7.0 \mathrm{~Hz}, 1 \mathrm{H}, \mathrm{CHCH}_{3}\right), 2.82-2.65$ $(\mathrm{m}, 2 \mathrm{H}$, isoindole $\mathrm{H}-3 \mathrm{a}, 7 \mathrm{a}), 1.85-1.64(\mathrm{~m}, 4 \mathrm{H}$, isoindole $\left.\mathrm{H}-4,4^{\mathrm{c}}, 7,7^{\mathrm{c}}\right), 1.58 / 1.51\left(\mathrm{~d}, J=7.1 \mathrm{~Hz}, 3 \mathrm{H}, \mathrm{CHCH}_{3}\right), 1.47-$ $1.25\left(\mathrm{~m}, 4 \mathrm{H}\right.$, isoindole $\left.\mathrm{H}-5,5 ; 6,6^{\circ}\right)$; LC-MS: $m / z=367$ $[\mathrm{M}]^{+}$; Anal. Calcd. for $\mathrm{C}_{19} \mathrm{H}_{21} \mathrm{~N}_{5} \mathrm{O}_{3}$ : C, 62.11; H, 5.76; N, 19.06. Found: C, 62.16; H, 5.82; N, 19.13 .

4-((1,3-Dioxooctahydro-2H-isoindole-2-yl)methyl)- $N^{\prime}-$ (quinazoline-4(3H)-ylidene)benzohydrazide (3b). Yield: 72.6\%; m.p. $263-265{ }^{\circ} \mathrm{C}$; IR $\left(\mathrm{cm}^{-1}\right): 3309,3180,3130$, 2918, 2953, 2854, 2779, 2714, 1698, 1612, 1525, 1471, $1374,1341,1310,1164,1093,906,762,690,662,646 ;{ }^{1} \mathrm{H}$ NMR $\delta$ (ppm): 11.51 (s, 1H, 3-NH), 10.52 (s, $1 \mathrm{H}, \mathrm{NHCO})$, 9.01-6.44 (m, 9H, H-2,5,6,7,8, Ar H-2,3,5,6), 4.62 (s, 2H, $\left.-\mathrm{CH}_{2^{-}}\right), 2.98-2.89(\mathrm{~m}, 2 \mathrm{H}$, isoindole $\mathrm{H}-3 \mathrm{a}, 7 \mathrm{a}), 1.90-1.58$ (m, $4 \mathrm{H}$, isoindole $\left.\mathrm{H}-4,4^{\mathrm{c}}, 7,7^{\circ}\right), 1.50-1.29(\mathrm{~m}, 4 \mathrm{H}$, isoindole H-5,5'6,6 ); LC-MS: $m / z=430$ [M+1], $431[\mathrm{M}+2]$; Anal. Calcd. for $\mathrm{C}_{24} \mathrm{H}_{23} \mathrm{~N}_{5} \mathrm{O}_{3}: \mathrm{C}, 67.12 ; \mathrm{H}, 5.40 ; \mathrm{N}, 16.31$. Found: C, 67.19; H, 5.48; N, 16.38 .

4-(1,3-Dioxooctahydro-2H-isoindole-2-yl)-N'-(quinazoline-4(3H)-ylidene)benzohydrazide (3c). Yield: 84.3\%; m.p. $187-190{ }^{\circ} \mathrm{C}$; IR $\left(\mathrm{cm}^{-1}\right)$ : 3421, 3280, 2993, 2913, 2855, $1698,1626,1537,1493,1475,1385,1167,1129,759,687$, $615 ;{ }^{1} \mathrm{H}$ NMR $\delta$ (ppm): 11.60 (s, 1H, 3-NH), 10.65 (s, 1H, NHCO), 8.58-6.95 (m, 9H, H-2,5,6,7,8, Ar H-2,3,5,6), $3.15-3.08(\mathrm{~m}, 2 \mathrm{H}$, isoindole $\mathrm{H}-3 \mathrm{a}, 7 \mathrm{a}), 1.97-1.70(\mathrm{~m}$, $4 \mathrm{H}$, isoindole $\left.\mathrm{H}-4,4^{4}, 7,7^{\mathrm{c}}\right), 1.57-1.35(\mathrm{~m}, 4 \mathrm{H}$, isoindole H-5,5,6,6); LC-MS: $m / z=416$ [M+1], 417 [M+2]; Anal. Calcd. for $\mathrm{C}_{23} \mathrm{H}_{21} \mathrm{~N}_{5} \mathrm{O}_{3}$ : C, 66.49; $\mathrm{H}, 5.10 ; \mathrm{N}, 16.86$. Found: C, 66.54; H, 5.16; N, 16.93 .

2-(1,3-Dioxo-1,3,3a,4,7,7a-hexahydro-2H-isoindole-2yl)-N'-(quinazoline-4(3H)-ylidene)propanehydrazide (3d). Yield: $71.8 \%$; m.p. $201-203{ }^{\circ} \mathrm{C}$, IR $\left(\mathrm{cm}^{-1}\right)$ : 3289, $3214,3069,3012,2960,2910,2886,2850,2780,2683$, $2410,1702,1603,1566,1466,1420,1383,1325,1198$, $1117,927,776,755,685,661 ;{ }^{1} \mathrm{H}$ NMR $\delta$ (ppm): 11.49 (s, $1 \mathrm{H}, 3-\mathrm{NH}), 10.09 / 10.00(\mathrm{~s}, 1 \mathrm{H}, \mathrm{NHCO}), 7.81-7.65(\mathrm{~m}, 2 \mathrm{H}$, 
$\mathrm{H}-2,5), 7.49$ (t, $J=7.8 \mathrm{~Hz}, 1 \mathrm{H}, \mathrm{H}-7), 7.20(\mathrm{t}, J=7.8 \mathrm{~Hz}, 1 \mathrm{H}$, H-6), $7.11(\mathrm{~d}, J=8.4 \mathrm{~Hz}, 1 \mathrm{H}, \mathrm{H}-8), 5.89$ (m, 2H, isoindole $\mathrm{H}-5,6), 5.13 / 4.71$ (q, $J=7.9,7.2 \mathrm{~Hz}, 1 \mathrm{H}, \mathrm{CHCH}_{3}$ ), 3.05$2.90(\mathrm{~m}, 2 \mathrm{H}$, isoindole $\mathrm{H}-3 \mathrm{a}, 7 \mathrm{a}), 2.46-2.01(\mathrm{~m}, 4 \mathrm{H}$, isoindole $\left.\mathrm{H}-4,4^{\prime}, 7,7^{`}\right), 1.44$ (d, $J=7.1 \mathrm{~Hz}, 3 \mathrm{H}, \mathrm{CHCH}_{3}$ ); LC-MS: $m / z=368[\mathrm{M}+3]$; Anal. Calcd. for $\mathrm{C}_{19} \mathrm{H}_{19} \mathrm{~N}_{5} \mathrm{O}_{3}: \mathrm{C}, 62.46$; H, 5.24; N, 19.17. Found: C, 62.49; H, 5.28; N, 19.23.

3-(1,3-Dioxo-1,3,3a,4,7,7a-hexahydro-2H-isoindole-2yl)-N'-(quinazoline-4(3H)-ylidene)propanehydrazide (3e). Yield: 68.4\%; m.p. $135-137^{\circ} \mathrm{C}$, IR $\left(\mathrm{cm}^{-1}\right)$ : 3489, 3409, $3343,3230,3128,3106,3078,3010,2988,2939,2859$, 2699, 1693, 1611, 1527, 1471, 1440, 1379, 1106, 755, 687; ${ }^{1} \mathrm{H}$ NMR $\delta$ (ppm): 11.53 (s, 1H, 3-NH), 10.03/9.52 (s, 1H, NHCO), 7.88 (d, J = 7.2 Hz, 1H, H-5), 7.74 (s, 1H, H-2), $7.37(\mathrm{t}, J=7.3 \mathrm{~Hz}, 1 \mathrm{H}, \mathrm{H}-7), 7.20$ (t, $J=7.5 \mathrm{~Hz}, 1 \mathrm{H}, \mathrm{H}-6)$, $7.13(\mathrm{~d}, J=7.8 \mathrm{~Hz}, 1 \mathrm{H}, \mathrm{H}-5), 5.95-5.76(\mathrm{~m}, 2 \mathrm{H}$, isoindole $\mathrm{H}-5,6), 3.67\left(\mathrm{~m}, 2 \mathrm{H}, \mathrm{CH}_{2} \mathrm{CH}_{2} \mathrm{~N}\right), 3.24-3.08$ (m, 2H, isoindole $3 \mathrm{a}, 7 \mathrm{a}), 2.82\left(\mathrm{t}, \mathrm{J}=7.5 \mathrm{~Hz}, 2 \mathrm{H}, \mathrm{CH}_{2} \mathrm{CH}_{2} \mathrm{~N}\right), 2.48-2.11$ ( $\mathrm{m}, 4 \mathrm{H}$, isoindole $\mathrm{H}-4,4^{\mathrm{c}}, 7,7^{\mathrm{c}}$ ); LC-MS: $m / z=366[\mathrm{M}+1]$, $367[\mathrm{M}+2]$; Anal. Calcd. for $\mathrm{C}_{19} \mathrm{H}_{19} \mathrm{~N}_{5} \mathrm{O}_{3}$ : C, 62.46; $\mathrm{H}$, 5.24; N, 19.17. Found: C, 62.43; H, 5.18; N, 19.13.

4-(1,3-Dioxo-1,3,3a,4,7,7a-hexahydro-2H-isoindole-2yl)-N'-(quinazoline-4(3H)-ylidene)benzohydrazide (3f). Yield: $94.3 \%$; m.p. $261-263{ }^{\circ} \mathrm{C}$, IR $\left(\mathrm{cm}^{-1}\right)$ : 3418, 3311, 3205, 3168, 3120, 3089, 3056, 3023, 2979, 2915, 2850, 1704, $1612,1536,1495,1474,1445,1386,1177,845,759,687$, 625; ${ }^{1} \mathrm{H}$ NMR $\delta(\mathrm{ppm}): 11.58(\mathrm{~s}, 1 \mathrm{H}, 3-\mathrm{NH}), 10.65 / 9.99$ (s, 1H, NHCO), 8.55-7.03 (m, 9H, H-2,5,6,7,8, Ar $\mathrm{H}-2,3,5,6), 6.15-5.81(\mathrm{~m}, 2 \mathrm{H}$, isoindole H-5,6), 3.39-3.19 (m, 2H, isoindole $\mathrm{H}-3 \mathrm{a}, 7 \mathrm{a}), 2.69-2.20(\mathrm{~m}, 4 \mathrm{H}$, isoindole H-4,4,7,7); LC-MS: $m / z=414[\mathrm{M}+1], 415$ [M+2]; Anal. Calcd. for $\mathrm{C}_{23} \mathrm{H}_{19} \mathrm{~N}_{5} \mathrm{O}_{3}$ : C, 66.82; $\mathrm{H}, 4.63 ; \mathrm{N}, 16.94$. Found: C, 66.89; H, 4.70; N, 17.01.

2-(1,3-Dioxo-1,3,3a,4,7,7a-hexahydro-2H-4,7-methanoisoindole-2-yl)-N'-(quinazoline-4(3H)-ylidene)acetohydrazide (3g). Yield: 71.6\%; m.p. $168-170{ }^{\circ} \mathrm{C}, \mathrm{IR}\left(\mathrm{cm}^{-}\right.$ $\left.{ }^{1}\right)$ : 3462, 3292, 3208, 3168, 3133, 3089, 3054, 3029, 2994, 2904, 2858, 2783, 1728, 1632, 1539, 1489, 1477, 1439, 1391, 1173, 845, 761, 683, 629; ${ }^{1} \mathrm{H}$ NMR $\delta$ (ppm): 11.51 (s, $1 \mathrm{H}, 3-\mathrm{NH}), 10.27 / 9.66$ (s, 1H, -NHCO-), 7.90 (d, $J=7.2$ $\mathrm{Hz}, 1 \mathrm{H}, \mathrm{H}-\mathrm{H}-5), 7.80$ (s, 1H, H-2), 7.40 (t, J=7.3 Hz, $1 \mathrm{H}$, $\mathrm{H}-7), 7.19$ (t, $J=7.4 \mathrm{~Hz}, 1 \mathrm{H}, \mathrm{H}-6), 7.13$ (d, $J=8.0 \mathrm{~Hz}, 1 \mathrm{H}$, $\mathrm{H}-8), 6.18-6.07$ ( $\mathrm{m}, 2 \mathrm{H}$, isoindole $\mathrm{H}-5,6), 4.73 / 4.37$ (s, 2H, $\left.\mathrm{CH}_{2}\right), 3.50-3.38(\mathrm{~m}, 2 \mathrm{H}$, isoindole $\mathrm{H}-3 \mathrm{a}, 7 \mathrm{a}), 3.39-3.27(\mathrm{~m}$, $2 \mathrm{H}$, isoindole $\mathrm{H}-4,7), 1.76-1.55\left(\mathrm{~m}, 2 \mathrm{H}\right.$, isoindole $\left.\mathrm{H}-8,8^{\mathrm{c}}\right)$; LC-MS: $m / z=364$ [M+1], $365[\mathrm{M}+2]$; Anal. Calcd. for $\mathrm{C}_{19} \mathrm{H}_{17} \mathrm{~N}_{5} \mathrm{O}_{3}$ : C, 62.80; $\mathrm{H}, 4.72 ; \mathrm{N}, 19.27$. Found: C, 62.87; $\mathrm{H}, 4.77$; N, 19.34 .

4-(1,3-Dioxo-1,3,3a,4,7,7a-hexahydro-2H-4,7-methanoisoindole-2-yl)methyl)-N'-(quinazoline-4-yl)benzohydrazide (3h). Yield: $88.1 \%$; m.p. $265-267^{\circ} \mathrm{C}$; IR $\left(\mathrm{cm}^{-1}\right)$ : 3376, 3060, 2990, 2962, 2914, 2849, 2690, 1695, 1611, 1525,
1471, 1375, 1339, 1167, 1093, 907, 763, 722, 690, 659, 644; ${ }^{1} \mathrm{H}$ NMR $\delta$ (ppm): 11.43 (s, 1H, 3-NH), 10.55 (s, 1H, s, 1H, -NHCO), 8.18 (d, J=7.4 Hz, 1H, H-5), 7.94-7.72 (m, 3H, $\mathrm{H}-2$, Ph H-2,6), 7.55 (t, $J=7.3 \mathrm{~Hz}, 1 \mathrm{H}, \mathrm{H}-7), 7.44-7.16$ (m, $4 \mathrm{H}, \mathrm{H}-6,8, \mathrm{Ph} \mathrm{H}-3,5), 6.04-5.86(\mathrm{~m}, 2 \mathrm{H}$, isoindole $\mathrm{H}-5,6)$, $4.46\left(\mathrm{~s}, 2 \mathrm{H},-\mathrm{CH}_{2}-\right), 3.42-3.34(\mathrm{~m}, 2 \mathrm{H}$, isoindole $\mathrm{H}-3 \mathrm{a}, 7 \mathrm{a})$, 3.34-3.29 (m, 2H, isoindole $\mathrm{H}-4,7), 1.74-1.48(\mathrm{~m}, 2 \mathrm{H}$, isoindole $\mathrm{H}-8,8^{\circ}$ ); LC-MS: $m / z=440[\mathrm{M}+1], 441[\mathrm{M}+2]$; Anal. Calcd. for $\mathrm{C}_{25} \mathrm{H}_{21} \mathrm{~N}_{5} \mathrm{O}_{5}$ : C, 68.33; H, 4.82; N, 15.94 . Found: C, 68.41; H, 4.88; N, 16.01.

4-(1,3-Dioxo-1,3,3a,4,7,7a-hexahydro-2H-4,7-methanoisoindole-2-yl)-N'-(quinazoline-4-yl)benzohydrazide (3i). Yield: $99.9 \%$; m.p. $187-189^{\circ} \mathrm{C}$; IR $\left(\mathrm{cm}^{-1}\right)$ : 3476,3310 , 3284, 3028, 2982, 2929, 2856, 2780, 1693, 1614, 1548, 1495, 1474, 1389, 1185, 760, 744, 721, 688, 615; ${ }^{1} \mathrm{H}$ NMR $\delta$ (ppm): 10.63 (s, -NHCO-), 8.21 (d, J= 7.2 Hz, 1H, H-5), 8.03-7.89 (m, 3H, H-2, Ph H-2,6), 7.59 (t, $J=7.4 \mathrm{~Hz}, 1 \mathrm{H}$, $\mathrm{H}-7$ ), 7.50-7.34 (m, 2H, H-6,8), 7.26 (d, $J=7.9 \mathrm{~Hz}, 2 \mathrm{H}$, $\mathrm{Ph} \mathrm{H}-3,5), 6.31-6.19$ (m, 2H, isoindole H-5,6), 3.60-3.48 ( $\mathrm{m}, 2 \mathrm{H}$, isoindole $\mathrm{H}-7 \mathrm{a}, 3 \mathrm{a}), 3.46-3.28(\mathrm{~m}, 2 \mathrm{H}$, isoindole $\mathrm{H}-4,7), 1.82-1.54\left(\mathrm{~m}, 2 \mathrm{H}\right.$, isoindole $\left.\mathrm{H}-8,8^{\circ}\right)$; LC-MS: $m / z$ $=426[\mathrm{M}+1], 428[\mathrm{M}+3]$; Anal. Calcd. for $\mathrm{C}_{24} \mathrm{H}_{19} \mathrm{~N}_{5} \mathrm{O}_{3}: \mathrm{C}$, 67.76; H, 4.50; N, 16.46. Found: C, 67.83; H, 4.58; N, 16.53.

The synthesized compounds are yellow, soluble in DMF, DMSO, dioxane, slightly soluble in alcohol, insoluble in water.

General Procedure for the Synthesis of 2-([1,2,4]Triazolo[1,5-c] quinazoline-2-yl-)alkyl-(alkylaryl-,aryl-) hydroisoindole-1,3(2H)-diones $4 \mathrm{a}-\mathrm{i}$

$0.01 \mathrm{~mol}$ of the corresponding hydrazides $\mathbf{3 a}-\mathbf{i}$ was dissolved in $25 \mathrm{~mL}$ of glacial acetic acid and was refluxed for 6 hours. The mixture was cooled. In the case of the residue formation it was filtered off. In the case of a solution, acetic acid was removed by distillation under vacuum. The resulting residue was stirred with a mixture of methanol and water and the precipitate was filtered. If necessary, the crude product can be crystallized.

(2-(1-([1,2,4]Triazolo[1,5-c]quinazoline-2-yl)ethyl) hexahydro-1H-isoindole-1,3(2H)-dione (4a). Yield: 62.9\%; m.p. $200-203{ }^{\circ} \mathrm{C}$; IR $\left(\mathrm{cm}^{-1}\right)$ : 2922, 2843, 2690, $2359,1705,1381,1342,1195,899,770 ;{ }^{1} \mathrm{H}$ NMR $\delta(\mathrm{ppm})$ : 9.40 (s, 1H, H-5), 8.39 (d, J = 7.6 Hz, 1H, H-10), 8.03 (d, $J=7.9 \mathrm{~Hz}, 1 \mathrm{H}, \mathrm{H}-7), 7.88(\mathrm{t}, J=8.1 \mathrm{~Hz}, 1 \mathrm{H}, \mathrm{H}-8), 7.76$ ( $\mathrm{t}, J=7.3 \mathrm{~Hz}, 1 \mathrm{H}, \mathrm{H}-9), 5.49$ (q, $J=6.7 \mathrm{~Hz}, 1 \mathrm{H}, \mathrm{CHCH}_{3}$ ), 3.01-2.86 (m, 2H, isoindole $3 \mathrm{a}, 7 \mathrm{a}), 1.89(\mathrm{~d}, J=7.0 \mathrm{~Hz}, 3 \mathrm{H}$, $\left.\mathrm{CHCH}_{3}\right), 1.86-1.69\left(\mathrm{~m}, 4 \mathrm{H}\right.$, isoindole $\left.\mathrm{H}-4,4^{\circ}, 7,7^{\mathrm{c}}\right), 1.59-$ $1.23\left(\mathrm{~m}, 4 \mathrm{H}\right.$, isoindole $\left.\mathrm{H}-5,5 ; 6,6^{\mathrm{c}}\right) ;{ }^{13} \mathrm{C} \mathrm{NMR} \delta(\mathrm{ppm})$ : 179.41/179.29 (C-1,3 isoindole), 166.16 (C-2l), 150.74 (C5), 142.69 (C-10b), 139.27 (C-6a), 132.80 (C-8), 129.63 (C-7), 128.99 (C-9), 123.46 (C-10), 117.76 (C-10a), 44.28 $\left(-\mathrm{CHCH}_{3}\right), 40.35$ (C-3a,7a isoindole), 23.44/23.36 (C-4,7 isoindole), 21.53/21.48 (C-5,6 isoindole), $15.82\left(\mathrm{CH}_{3}\right)$; LC-MS: $m / z=350[\mathrm{M}+1], 352[\mathrm{M}+2]$; Anal. Calcd. for 
$\mathrm{C}_{19} \mathrm{H}_{19} \mathrm{~N}_{5} \mathrm{O}_{2}$ : C, 65.32; H, 5.48; N, 20.04. Found: C, 65.39; $\mathrm{H}, 5.52 ; \mathrm{N}, 20.11$.

2-(4-([1,2,4]Triazolo[1,5-c]quinazoline-2-yl)benzyl) hexahydro-1H-isoindole-1,3(2H)-dione (4b). Yield: 55.6\%; m.p. $248-250{ }^{\circ} \mathrm{C}$; IR $\left(\mathrm{cm}^{-1}\right)$ : 2957, 2929, 2854, $2456,1689,1479,1423,1397,1334,1165,899,775,742 ;{ }^{1} \mathrm{H}$ NMR $\delta$ (ppm): $9.48(\mathrm{~s}, 1 \mathrm{H}, \mathrm{H}-5), 8.55(\mathrm{~d}, J=7.7 \mathrm{~Hz}, 1 \mathrm{H}$, $\mathrm{H}-10), 8.26$ (d, $J=8.1 \mathrm{~Hz}, 2 \mathrm{H}$, Ar H-2,6), 8.06 (d, $J=8.4$ $\mathrm{Hz}, 1 \mathrm{H}, \mathrm{H}-7), 7.90$ (t, $J=7.9 \mathrm{~Hz}, 1 \mathrm{H}, \mathrm{H}-8), 7.81$ (t, $J=7.6$ $\mathrm{Hz}, 1 \mathrm{H}, \mathrm{H}-9$ ), 7.45 (d, $J=7.9 \mathrm{~Hz}, 2 \mathrm{H}, \mathrm{Ar} \mathrm{H}-3,5), 4.64$ (s, $\left.2 \mathrm{H}, \mathrm{CH}_{2}\right), 2.98-2.93(\mathrm{~m}, 2 \mathrm{H}$, isoindole $3 \mathrm{a}, 7 \mathrm{a}), 1.87-1.57$ (m, $4 \mathrm{H}$, isoindole $\left.\mathrm{H}-4,4,7,7^{\mathrm{c}}\right), 1.54-1.26(\mathrm{~m}, 4 \mathrm{H}$, isoindole H-5,5'6,6 ); LC-MS: $m / z=412$ [M+1], 413 [M+2]; Anal. Calcd. for $\mathrm{C}_{24} \mathrm{H}_{21} \mathrm{~N}_{5} \mathrm{O}_{2}: \mathrm{C}, 70.06 ; \mathrm{H}, 5.14 ; \mathrm{N}, 17.02$. Found: C, 70.12; H, 5.18; N, 17.11 .

2-(4-([1,2,4]Triazolo[1,5-c]quinazoline-2-yl)phenyl)hexa hydro-1H-isoindole-1,3(2H)-dione (4c). Yield: 74.6\%; m.p. $>300{ }^{\circ} \mathrm{C}$; IR $\left(\mathrm{cm}^{-1}\right): 2989,2924,2863,1701,1514$, $1477,1445,1357,1164,1122,899,769,749,713,628$; ${ }^{1} \mathrm{H}$ NMR $\delta$ (ppm): 9.50 (s, 1H, H-5), 8.57 (d, $J=7.9 \mathrm{~Hz}$, $1 \mathrm{H}, \mathrm{H}-10), 8.41$ (d, $J=8.4 \mathrm{~Hz}, 2 \mathrm{H}, \mathrm{Ar} \mathrm{H}-2,6), 8.07$ (d, $J$ $=8.2 \mathrm{~Hz}, 1 \mathrm{H}, \mathrm{H}-7), 7.96(\mathrm{t}, J=7.6 \mathrm{~Hz}, 1 \mathrm{H}, \mathrm{H}-8), 7.82(\mathrm{t}$, $J=7.5 \mathrm{~Hz}, 1 \mathrm{H}, \mathrm{H}-9), 7.49$ (d, $J=8.4 \mathrm{~Hz}, 2 \mathrm{H}, \mathrm{Ar} \mathrm{H}-3,5)$, $3.13(\mathrm{~m}, 2 \mathrm{H}$, isoindole $3 \mathrm{a}, 7 \mathrm{a}), 1.97-1.72(\mathrm{~m}, 4 \mathrm{H}$, isoindole $\left.\mathrm{H}-4,4^{`}, 7,7^{\mathrm{c}}\right), 1.57-1.39\left(\mathrm{~m}, 4 \mathrm{H}\right.$, isoindole $\left.\mathrm{H}-5,5^{`}, 6,6^{\prime}\right)$; LC-MS: $m / z=398[\mathrm{M}+1], 400[\mathrm{M}+2]$; Anal. Calcd. for $\mathrm{C}_{23} \mathrm{H}_{19} \mathrm{~N}_{5} \mathrm{O}_{2}$ : C, 69.51; H, 4.82; N, 17.62. Found: C, 69.58; $\mathrm{H}, 4.91 ; \mathrm{N}, 17.71$.

2-(1-([1,2,4]Triazolo[1,5-c]quinazoline-2-yl)ethyl)-3a, 4,7,7a-tetrahydro-1H-isoindole-1,3(2H)-dione (4d). Yield: $71.2 \%$; m.p. $180-184^{\circ} \mathrm{C}$; IR $\left(\mathrm{cm}^{-1}\right): 2954,2899,1701$, $1493,1387,1360,1214,900,773,706 ;{ }^{1} \mathrm{H}$ NMR $\delta$ (ppm): 9.39 (s, 1H, H-5), 8.41 (d, $J=7.8 \mathrm{~Hz}, 1 \mathrm{H}, \mathrm{H}-10), 8.02$ (d, $J=8.3 \mathrm{~Hz}, 1 \mathrm{H}, \mathrm{H}-7), 7.87(\mathrm{t}, J=7.6 \mathrm{~Hz}, 1 \mathrm{H}, \mathrm{H}-8), 7.76(\mathrm{t}$, $J=7.5 \mathrm{~Hz}, 1 \mathrm{H}, \mathrm{H}-9), 5.93(\mathrm{~m}, 2 \mathrm{H}$, isoindole $\mathrm{H}-5,6), 5.47$ (q, $\left.J=7.1 \mathrm{~Hz}, 1 \mathrm{H}, \mathrm{CHCH}_{3}\right), 3.38-3.09(\mathrm{~m}, 2 \mathrm{H}$, isoindole 3a,7a), 2.58-2.49 (m, $2 \mathrm{H}$, isoindole $\mathrm{H}-4,7), 2.37-2.15$ (m, $2 \mathrm{H}$, isoindole $\left.\mathrm{H}-4^{c}, 7^{c}\right), 1.83\left(\mathrm{~d}, J=7.1 \mathrm{~Hz}, 3 \mathrm{H}, \mathrm{CHCH}_{3}\right)$; LC-MS: $m / z=348[\mathrm{M}+1], 349$ [M+2]; Anal. Calcd. for $\mathrm{C}_{19} \mathrm{H}_{17} \mathrm{~N}_{5} \mathrm{O}_{2}$ : C, 65.69; H, 4.93; N, 20.16. Found: $\mathrm{C}, 65.66$; $\mathrm{H}, 4.98 ; \mathrm{N}, 20.13$.

\section{2-(2-([1,2,4]Triazolo[1,5-c]quinazoline-2-yl)ethyl)-3a,} 4,7,7a-tetrahydro-1H-isoindole-1,3(2H)-dione (4e). Yield: $40.0 \%$; m.p. $165-167{ }^{\circ} \mathrm{C}$; IR $\left(\mathrm{cm}^{-1}\right)$ : 2982,2843 , 2828, 2712, 1693, 1399, 1362, 1325, 1211, 1157, 1010, 929, 904, 770, 698; ${ }^{1} \mathrm{H}$ NMR $\delta$ (ppm): 9.37 (s, $\left.1 \mathrm{H}, \mathrm{H}-5\right)$, $8.43(\mathrm{~d}, J=7.6 \mathrm{~Hz}, 1 \mathrm{H}, \mathrm{H}-10), 8.03(\mathrm{~d}, J=8.3 \mathrm{~Hz}, 1 \mathrm{H}$, $\mathrm{H}-7), 7.88(\mathrm{t}, J=7.9 \mathrm{~Hz}, 1 \mathrm{H}, \mathrm{H}-8), 7.77(\mathrm{t}, J=7.5 \mathrm{~Hz}, 1 \mathrm{H}$, $\mathrm{H}-9)$, 5.94-5.66 (m, 2H, isoindole $\mathrm{H}-5,6), 3.85(\mathrm{t}, J=7.3$ $\left.\mathrm{Hz}, 2 \mathrm{H}, \mathrm{CH}_{2} \mathrm{CH}_{2} \mathrm{~N}\right), 3.20-2.97\left(\mathrm{~m}, 4 \mathrm{H}, \mathrm{CH}_{2} \mathrm{CH}_{2} \mathrm{~N}\right.$, isoindole $3 \mathrm{a}, 7 \mathrm{a}), 2.50-2.40(\mathrm{~m}, 2 \mathrm{H}$, isoindole $\mathrm{H}-4,7), 2.32-2.06$ ( $\mathrm{m}, 2 \mathrm{H}$, isoindole $\left.\mathrm{H}-4^{\natural}, 7^{\mathrm{c}}\right)$; LC-MS: $m / z=348[\mathrm{M}+1], 349$
[M+2]; Anal. Calcd. for $\mathrm{C}_{19} \mathrm{H}_{17} \mathrm{~N}_{5} \mathrm{O}_{2}$ : C, 65.69; $\mathrm{H}, 4.93 ; \mathrm{N}$, 20.16. Found: C, 65.74; H, 5.02; N, 20.23.

2-(4-([1,2,4]Triazolo[1,5-c]quinazoline-2-yl)phenyl)3a,4,7,7a-tetrahydro-1H-isoindole-1,3(2H)-dione (4f). Yield: $82.25 \%$; m.p. $250-253{ }^{\circ} \mathrm{C}$; IR $\left(\mathrm{cm}^{-1}\right): 1703,1514$, $1478,1446,1360,1315,1177,899,842,751,717,672 ;{ }^{1} \mathrm{H}$ NMR $\delta$ (ppm): $9.50(\mathrm{~s}, 1 \mathrm{H}, \mathrm{H}-5), 8.56(\mathrm{~d}, J=8.3 \mathrm{~Hz}, 1 \mathrm{H}$, $\mathrm{H}-10$ ), 8.39 (d, $J=8.3 \mathrm{~Hz}, 2 \mathrm{H}, \mathrm{Ar} \mathrm{H}-2,6), 8.20$ (d, $J=8.5$ $\mathrm{Hz}, 1 \mathrm{H}, \mathrm{H}-7), 8.06$ (t, $J=8.6 \mathrm{~Hz}, 1 \mathrm{H}, \mathrm{H}-8), 7.86$ (t, $J=8.6$ $\mathrm{Hz}, 1 \mathrm{H}, \mathrm{H}-9), 7.44$ (d, $J=8.4 \mathrm{~Hz}, 2 \mathrm{H}, \mathrm{Ar} \mathrm{H}-3,5), 6.05-$ $5.92(\mathrm{~m}, 2 \mathrm{H}$, isoindole $\mathrm{H}-5,6), 3.12-2.85(\mathrm{~m}, 2 \mathrm{H}$, isoindole $3 \mathrm{a}, 7 \mathrm{a}), 2.66-2.56(\mathrm{~m}, 2 \mathrm{H}$, isoindole $\mathrm{H}-4,7), 2.37-2.23$ (m, $2 \mathrm{H}$, isoindole $\mathrm{H}-4^{\mathrm{c}}, 7^{\mathrm{c}}$ ); LC-MS: $m / z=396[\mathrm{M}+1], 397$ $[\mathrm{M}+2]$; Anal. Calcd. for $\mathrm{C}_{23} \mathrm{H}_{17} \mathrm{~N}_{5} \mathrm{O}_{2}: \mathrm{C}, 69.86 ; \mathrm{H}, 4.33 ; \mathrm{N}$, 17.71. Found: C, 69.94; H, 4.41; N, 17.80 .

2-([1,2,4]Triazolo[1,5-c]quinazoline-2-ylmethyl)-3a, 4,7,7a-tetrahydro-1H-4,7-methanoisoindole-1,3 (2H)-dione (4g). Yield: $85.8 \%$; m.p. $215-217^{\circ} \mathrm{C}$; IR $\left(\mathrm{cm}^{-1}\right)$ : 2994, 2863, 2812, 1721, 1514, 1475, 1446, 1368, 1323, $1183,899,846,751,721,682$; ${ }^{1} \mathrm{H}$ NMR $\delta$ (ppm): 9.40 (s, $1 \mathrm{H}, \mathrm{H}-5), 8.43$ (d, $J=7.6 \mathrm{~Hz}, 1 \mathrm{H}, \mathrm{H}-10), 8.03$ (d, $J=7.6$ $\mathrm{Hz}, 1 \mathrm{H}, \mathrm{H}-7), 7.89$ (t, $J=7.5 \mathrm{~Hz}, 1 \mathrm{H}, \mathrm{H}-8), 7.78(\mathrm{t}, J=7.5$ $\mathrm{Hz}, 1 \mathrm{H}, \mathrm{H}-9), 6.15$ (s, 2H, isoindole $\mathrm{H}-5,6), 4.73$ (s, 2H, $\left.\mathrm{NCH}_{2}\right), 3.46(\mathrm{~s}, 2 \mathrm{H}$, isoindole $\mathrm{H}-4,7), 3.37(\mathrm{~s}, 2 \mathrm{H}$, isoindole $\mathrm{H}-3 \mathrm{a}, 7 \mathrm{a}), 1.67$ (dd, $J=20.6,8.0 \mathrm{~Hz}, 2 \mathrm{H}$, isoindole $\mathrm{H}-8,8^{\mathrm{c}}$ ); LC-MS: $m / z=346[\mathrm{M}+1], 348[\mathrm{M}+3]$; Anal. Calcd. for $\mathrm{C}_{19} \mathrm{H}_{15} \mathrm{~N}_{5} \mathrm{O}_{2}$ : C, 66.08; H, 4.38; N, 20.28. Found: C, 66.17; $\mathrm{H}, 4.46 ; \mathrm{N}, 20.32$.

2-(4-([1,2,4]Triazolo[1,5-c]quinazoline-2-yl)benzyl)-3a,4,7,7a-tetrahydro-1 H-4,7-methanoisoindole-1,3(2H)-dione (4h). Yield: 33.06\%; m.p. $219-221^{\circ} \mathrm{C}$; IR $\left(\mathrm{cm}^{-1}\right): 2996,2974,2918,1695,1515,1476,1420,1386$, $1334,1160,1118,899,769,756,727,714 ;{ }^{1} \mathrm{H} N M R \delta(\mathrm{ppm})$ : $9.48(\mathrm{~s}, 1 \mathrm{H}, \mathrm{H}-5), 8.56(\mathrm{~d}, J=8.2 \mathrm{~Hz}, 1 \mathrm{H}, \mathrm{H}-10), 8.24(\mathrm{~d}, J=$ $7.1 \mathrm{~Hz}, 2 \mathrm{H}, 1,4$-phenylene $\mathrm{H}-2,6), 8.06(\mathrm{~d}, J=8.2 \mathrm{~Hz}, 1 \mathrm{H}$, $\mathrm{H}-7), 7.91(\mathrm{t}, J=8.2 \mathrm{~Hz}, 1 \mathrm{H}, \mathrm{H}-8), 7.81(\mathrm{t}, J=8.2 \mathrm{~Hz}, 1 \mathrm{H}$, $\mathrm{H}-7, \mathrm{H}-9), 7.40$ (d, $J=7.2 \mathrm{~Hz}, 1 \mathrm{H}, 1,4$-phenylene $\mathrm{H}-3,4)$, 5.95 (s, isoindole $\mathrm{H}-5,6), 4.48\left(\mathrm{~s}, 2 \mathrm{H},-\mathrm{NCH}_{2^{-}}\right), 3.37$ (s, $2 \mathrm{H}$, isoindole $\mathrm{H}-4,7), 3.32(\mathrm{~s}, 2 \mathrm{H}$, isoindole $\mathrm{H}-3 \mathrm{a}, 7 \mathrm{a}), 1.63$ (dd, $J=22.0,8.5 \mathrm{~Hz}, 2 \mathrm{H}$, isoindole $\mathrm{H}-8,8^{\mathrm{c}}$ ); ${ }^{13} \mathrm{C}$ NMR $\delta$ (ppm): 177.75 (C-1,3; isoindole), 169.81 (C-2), 153.47 (C5), 143.95 (C-10b), 137.96 (C-6a), 135.40 (C-4; Ar), 134.92 (C-5,6; isoindole), 132.76 (C-8), 131.69 (C-1a; Ar), 129.55 (C-7), 129.01 (C-9), 128.90 (C-2,6; Ar), 127.51 (C-3,5; Ar), 123.81 (C-10), 114.13 (C-10a), 52.36 (C-8; isoindole), 45.87 (C-4,7; isoindole), 44.87 (C-3a,7a; isoindole), 41.49 (- $\left.\mathrm{NCH}_{2}{ }^{-}\right)$; LC-MS: $m / z=422[\mathrm{M}+1], 424[\mathrm{M}+3]$; Anal. Calcd. for $\mathrm{C}_{25} \mathrm{H}_{19} \mathrm{~N}_{5} \mathrm{O}_{2}: \mathrm{C}, 71.25 ; \mathrm{H}, 4.54 ; \mathrm{N}, 16.62$. Found: C, $71.31 ; \mathrm{H}, 4.58 ; \mathrm{N}, 16.68$.

2-(4-([1,2,4]Triazolo[1,5-c]quinazoline-2-yl)phenyl)-3a, 4, 7,7a-tetrahydro-1H-4,7-methanoisoindole-1,3(2H)-dione (4i). Yield: $86.87 \%$; m.p. $269-271{ }^{\circ} \mathrm{C}$; 
IR $\left(\mathrm{cm}^{-1}\right): 2982,2932,2861,2812,1703,1477,1447,1357$, $1186,899,838,778,744,708,669,608 ;{ }^{1} \mathrm{H}$ NMR $\delta$ (ppm): 9.50 (s, 1H, H-5), 8.57 (d, J= $7.7 \mathrm{~Hz}, 1 \mathrm{H}, \mathrm{H}-10), 8.36$ (d, $J$ $=7.8 \mathrm{~Hz}, 2 \mathrm{H}, 1,4$-phenylene $\mathrm{H}-2,6), 8.07(\mathrm{~d}, J=7.7 \mathrm{~Hz}, 1 \mathrm{H}$, $\mathrm{H}-7), 7.91(\mathrm{t}, J=7.7 \mathrm{~Hz}, 1 \mathrm{H}, \mathrm{H}-8), 7.82(\mathrm{t}, J=7.7 \mathrm{~Hz}, 1 \mathrm{H}$, $\mathrm{H}-9$ ), 7.32 (d, $J=7.7 \mathrm{~Hz}, 2 \mathrm{H}, 1,4$-phenylene $\mathrm{H}-3,5), 6.27$ (s, $2 \mathrm{H}$, isoindole $\mathrm{H}-5,6), 3.52$ (s, $2 \mathrm{H}$, isoindole $\mathrm{H}-4,7), 3.43$ (s, 2H, isoindole H-3a,7a), 1.59 (dd, $J=20.7,7.9 \mathrm{~Hz}, 2 \mathrm{H}$, isoindole H-8,8'); LC-MS: $m / z=408$ [M+1], 409 [M+2]; Anal. Calcd. for $\mathrm{C}_{24} \mathrm{H}_{17} \mathrm{~N}_{5} \mathrm{O}_{2}$ : C, 70.75; H, 4.21; N, 17.19. Found: C, 70.82; H, 4.28; N, 17.23.

The synthesized compounds are yellow, soluble in DMF, DMSO, dioxane, slightly soluble in alcohol, insoluble in water.

\section{Results and Discussion}

\section{1. Chemistry}

As it has been mentioned above, the combination in one molecule of more pharmacophore fragments, which are responsible for hypoglycemic effect, is interesting as well as offering further structural modifications and prospects to study such compounds in pharmacological screenings. Thus, following the search strategy, $\mathrm{N}$-carboxyalkyl-(arylalkyl-,aryl-)isoindoline-1,3-diones 1a-i were utilized in the reaction with $N, N^{\prime}$-carbonyldiimidazole for obtaining corresponding imidazolides, in the first stage of the study (Scheme 1). The last ones formed previously unknown hydrazides $\mathbf{3 a}$-i in the reaction with 4-hydrazinoquinazoline (2a). The presence of anhydrous dioxane and the temperature control (not above $80{ }^{\circ} \mathrm{C}$ ) is obligatory for the regioselectivity of the reaction. Reaction products $\mathbf{3 a}-\mathbf{i}$ are formed with high yields and in most cas- es they do not require additional purification. The appearance in the ${ }^{1} \mathrm{H}$ NMR spectrum of low-field broad singlet of exocyclic $\mathrm{NH}$-group proton of the quinazoline ring at the range of 11.60-11.46 ppm and $N H$-protons signal of the hydrazide fragment at the $10.65-9.52 \mathrm{ppm}$ indicates the 3a-i compounds formation. It is important to note, that some hydrazides, like $\mathbf{3 a}, \mathbf{3 d} \mathbf{- 3} \mathbf{g}$ are characterized by doubling of $\mathrm{NH}$-protons signals of the hydrazide fragment due to the prototropic tautomerism in DMSO, resulting in the appearance of aromatic and aliphatic protons in the form of multiplets or double signals. Besides, the IR spectra of hydrazides $3 \mathbf{a}-\mathbf{i}$ are characterized by $-\mathrm{C}=\mathrm{O}$ groups band valence vibrations of isoindole fragments at the 1728-1693 $\mathrm{cm}^{-1}$ and $-\mathrm{C}(\mathrm{O}) \mathrm{NH}$-groups (amide-I) at the 1632-1603 $\mathrm{cm}^{-1}$, valence-deformation vibrations of the N-H and C-N (amide-II) bonds at the $1566-1525 \mathrm{~cm}^{-1}$ as well as the band of $\mathrm{NH}$-groups valence vibrations at the range of the $3489-3168$ and $3180-2993 \mathrm{~cm}^{-1}$.

The formed hydrazides $\mathbf{3 a}-\mathbf{i}$ were subjected to cyclocondensation by known methods. ${ }^{29}$ As we expected, intermediate $[1,2,4]$ triazolo $[4,3-c]$ quinazolines are subjected to the recyclic isomerization by the Dimroth rearrangement under acidic catalysis conditions with 2-(2-([1,2,4]triazolo[1,5-c] quinazoline-2-yl)-alkyl-(arylalkyl-,aryl-)- $1 H$ isoindole-1,3(2H)-dione formation (4a-i, Scheme 1). ${ }^{29,30}$ Signals of triazine $[1,5-c]$ quinazoline ring protons, namely the low-field singlet of H-5 nucleus at the 9.50-9.37 ppm, doublet signals of ABCD-system at the 8.57-8.39 ppm (H10) and 8.20-8.03 ppm (H-7) and triplets at the 8.06-7.87 ppm (H-8) and 7.86-7.76 ppm (H-9) are typical for the ${ }^{1} \mathrm{H}$ NMR spectra of compounds $\mathbf{4 a}-\mathbf{i}$. It is important, that the aromatic proton signals of compounds $\mathbf{4 a - i}$, in comparison with compounds $\mathbf{3 a - i}$, undergo a significant paramagnetic shift that indicated the formation of an elec-
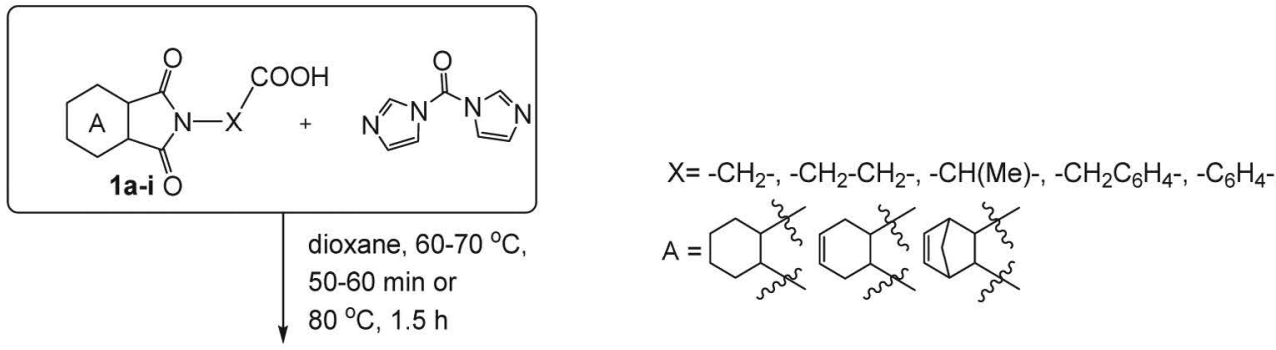<smiles>N/N=c1\[nH]cnc2ccccc12</smiles>

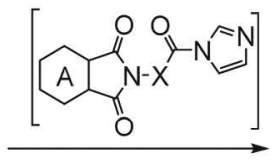

dioxane, $80^{\circ} \mathrm{C}, 1,5 \mathrm{~h}$<smiles>O=C(N/N=c1\[nH]cnc2ccccc12)N1C(=O)C2CCCCC2C1=O</smiles>
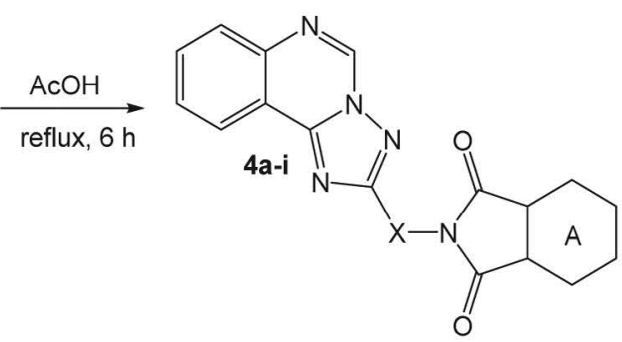

Scheme 1. The synthesis of $N$-carboxyalkyl-(arylalkyl-,aryl-)isoindoline-1,3-diones (3H-quinazoline-4-ylidene)hydrazides and hydrogenated 2-([1,2,4]triazolo[1,5-c]quinazoline-2-yl-)alkyl-(alkaryl-,aryl-)isoindole-1,3(2H)-diones. 
tron-deficient tricyclic system. In favor of compounds $\mathbf{4 a}$ and $4 \mathrm{~h}$ formation is also indicative the appearance of the characteristic signals of $s p^{2}$-hybridized C-2 atoms at the $169.81-166.16 \mathrm{ppm}$ and C-5 at the $153.47-150.74 \mathrm{ppm}$ of triazino[1,5-c] quinazoline ring in the ${ }^{13} \mathrm{C}$ NMR spectra. ${ }^{29}$

In addition, the ${ }^{1} \mathrm{H}$ NMR spectra of compounds $3 \mathbf{a}-$ i, $4 \mathbf{a}-\mathbf{i}$ are characterized by signals of the corresponding hydrogenated isoindoline moieties. Thus, a totally hydrogenated isoindole moiety of compounds $3 \mathbf{a}-\mathbf{3 c}$ and $\mathbf{4 a - 4 c}$ in the ${ }^{1} \mathrm{H}$ NMR spectra is observed as a set of multiplet signals of axial and equatorial protons at the 3.15-2.65 ppm (H-3a, H-7a), at the 1.97-1.58 ppm (H-4,4,7,7') and at the $1.59-1.23 \mathrm{ppm}\left(\mathrm{H}-5,5^{\prime}, 6,6^{\prime}\right)$. The hexahydroisoindole moiety (compounds $\mathbf{3 d}-\mathbf{3 f}, \mathbf{4 d - 4 f}$ ) is in the ${ }^{1} \mathrm{H}$ NMR spectra characterized by multiplet signals of $\mathrm{H}-5,6$ at the 6.15-5.66 ppm and a set of multiplets of axial and equatorial protons at the $3.24-2.90 \mathrm{ppm}(\mathrm{H}-3 \mathrm{a}, \mathrm{H}-7 \mathrm{a})$ and $2.69-2.01 \mathrm{ppm}\left(\mathrm{H}-4,4,7,7^{\prime}\right)$. Whereas, the hexahydro-4,7-methanoisoindole moiety (compounds $\mathbf{3 g}-\mathbf{3 i}$ ) is in the ${ }^{1} \mathrm{H}$ NMR spectra characterized by multiplet signal of H-5,6 at the $6.31-5.86 \mathrm{ppm}$ and a set of multiplet signals at the 3.60-3.34 ppm (H-3a, H-7a), 3.46-3.27 ppm $(\mathrm{H}-4,7)$ and $1.82-1.48 \mathrm{ppm}\left(\mathrm{H}-8,8^{\prime}\right)$. The chemical shifts and multiplicity of the indicated signals in the compounds 4g-4i differs slightly: the $\mathrm{H}-5,6$ signals are recorded at the 6.27-5.95 ppm, H-4,7 at the 3.52-3.37 ppm, H-3a, H-7a at the 3.43-3.32 ppm and a doublet of doublets for $\mathrm{H}-8,8^{\text {' }}$ appears at the 1.67-1.59 $\mathrm{ppm}$. In addition, there are signals of linker alkyl and aryl groups for which the corresponding multiplicity and chemical shift of protons are typical for the ${ }^{1} \mathrm{H}$ NMR spectra. ${ }^{31}$

In the IR spectra of compounds $4 \mathbf{a}-\mathbf{i}$ there are no bands of vibrations of exchangeable protons differing from compounds $\mathbf{3} \mathbf{a}-\mathbf{i}$, whereas the bands of $\mathrm{C}=\mathrm{O}$ groups of isoindole fragments at the $1721-1689 \mathrm{~cm}^{-1}$ and

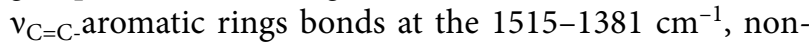
flat deformation vibrations of $\gamma_{=\mathrm{C}-\mathrm{H}-}$ bond at the 899-608 $\mathrm{cm}^{-1}$ and intensive bands of symmetric and antisymmetric valence vibrations of $-\mathrm{CH}_{2}$ groups at the 2989-2359 $\mathrm{cm}^{-1}$ are present.

\section{2. Hypoglycemic Assay for Preliminary in vivo Testing}

The screening results of hypoglycemic activity (Table 1) showed that synthesized compounds have different effects on glucose level in the blood of experimental animals. Thus, the compounds $\mathbf{3 a}, \mathbf{3 f}, \mathbf{3 h}, \mathbf{4 a}, \mathbf{4 c}$ and $\mathbf{4 f}$ decreased the glucose level on the second hour of the experiment on $13.99 \%, 4.09 \%, 11.86 \%, 18.47 \%, 3.18 \%$ and $8.61 \%$ respectively, substantially yielded to the action of «Metformine». It is important that only compounds $\mathbf{3 a}$, 3h, $\mathbf{4 f}$ keep this activity on the fourth hour of the experiment (reduced glucose level on $27.87 \%, 10.51 \%$ and $2.65 \%$ respectively). While the compounds $\mathbf{3 b}, \mathbf{3 c}, \mathbf{3 e}, \mathbf{3 i}, \mathbf{4 b}, \mathbf{4 e}$ and $4 \mathbf{i}$ under the given experimental conditions, on the contrary, increased glucose level on the second hour of the experiment from 4.89 to $15.79 \%$ (Table 1 ).

The insulinotropic effect of prandial glucose regulators comes very quickly unlike other oral hypoglycemic agents. Insulin secretion stimulation from $\beta$-cells of the pancreas islands is formed in 5-7 minutes (Mitiglinide, Nateglinide) or in 10-30 minutes after using (Repaglinide) these drugs in response to food intake. This is achieved by closing ATP-dependent potassium channels in the membrane of $\beta$-cells due to their depolarization. Molecular docking to KATP channel, PDB ID-6BAA was conducted, for a number of synthesized compounds $(\mathbf{3 a}, \mathbf{3 f}, \mathbf{3 h}, \mathbf{4 a}$,

Table 1 The synthesized compounds' influence on glucose level in blood of experimental animals (\%)

\begin{tabular}{|c|c|c|c|c|c|c|c|}
\hline Comp. & $\begin{array}{l}\text { Initial glucose } \\
\text { level }\end{array}$ & $\begin{array}{l}\text { Glucose level } \\
\text { in } 2 \mathrm{~h}\end{array}$ & $\%$ & $\begin{array}{l}\text { Glucose level } \\
\text { in } 4 \mathrm{~h}\end{array}$ & $\%$ & $\begin{array}{l}\text { Glucose level } \\
\text { in } 6 \mathrm{~h}\end{array}$ & $\%$ \\
\hline Intact group & $6.12+0.12$ & $6.60 \pm 0.08$ & +7.84 & $5.62 \pm 0.14$ & -8.17 & $5.85 \pm 0.13$ & -4.41 \\
\hline $3 \mathbf{a}$ & $7.93 \pm 0.83$ & $6.82 \pm 0.69$ & -13.99 & $5.72 \pm 0.49$ & -27.87 & $8.46 \pm 0.79$ & +6.68 \\
\hline $3 b$ & $6.46 \pm 0.12^{\mathrm{a}}$ & $6.94 \pm 0.34$ & +7.43 & $8.08 \pm 0.79$ & +25.08 & $8.28 \pm 0.95$ & +28.17 \\
\hline $3 c$ & $6.12 \pm 0.17^{\mathrm{a}}$ & $6.80 \pm 0.29$ & +11.11 & $7.30 \pm 0.74$ & +19.28 & $7.02 \pm 0.65$ & +14.70 \\
\hline $3 e$ & $6.68 \pm 0.96$ & $7.62 \pm 0.17^{\mathrm{a}}$ & +14.07 & $8.14 \pm 0.27$ & +21.86 & $8.04 \pm 0.15^{\mathrm{a}}$ & +20.36 \\
\hline $3 f$ & $5.38 \pm 0.35$ & $5.16 \pm 0.17^{\mathrm{a}}$ & -4.09 & $5.80 \pm 0.29$ & +7.81 & $5.42 \pm 0.29$ & +0.74 \\
\hline $3 h$ & $5.90 \pm 0.23^{\mathrm{a}}$ & $5.20 \pm 0.19$ & -11.86 & $5.28 \pm 0.21$ & -10.51 & $5.20 \pm 0.19^{\mathrm{a}}$ & -11.86 \\
\hline $3 \mathbf{i}$ & $6.56 \pm 0.05^{\mathrm{a}}$ & $7.20 \pm 0.17^{\mathrm{a}}$ & +9.75 & $7.72 \pm 0.07^{\mathrm{a}}$ & +17.68 & $7.72 \pm 0.20$ & +17.68 \\
\hline $4 a$ & $6.82 \pm 0.22$ & $5.56 \pm 0.27$ & -18.47 & $7.34 \pm 0.45$ & +7.62 & $6.88 \pm 0.17^{\mathrm{a}}$ & +0.88 \\
\hline $4 b$ & $6.12 \pm 0.23$ & $6.94 \pm 0.39$ & +13.39 & $7.08 \pm 0.47$ & +15.68 & $6.78 \pm 0.35$ & +10.78 \\
\hline $4 c$ & $6.28 \pm 0.34$ & $6.08 \pm 0.26$ & -3.18 & $6.56 \pm 0.33$ & +4.46 & $6.30 \pm 0.13^{\mathrm{a}}$ & +0.32 \\
\hline $4 e$ & $6.28 \pm 0.06^{\mathrm{a}}$ & $7.10 \pm 0.13^{\mathrm{a}}$ & +13.06 & $7.58 \pm 0.33$ & +20.70 & $8.36 \pm 0.41$ & +33.12 \\
\hline $4 \mathrm{f}$ & $6.04 \pm 0.20$ & $5.52 \pm 0.33$ & -8.61 & $5.88 \pm 0.17^{\mathrm{a}}$ & -2.65 & $5.34 \pm 0.21$ & -11.59 \\
\hline $4 h$ & $5.72 \pm 0.41$ & $6.00 \pm 0.28$ & +4.89 & $5.16 \pm 0.35$ & -9.79 & $4.98 \pm 0.37$ & -12.94 \\
\hline $4 \mathbf{i}$ & $6.46 \pm 0.07^{\mathrm{a}}$ & $7.48 \pm 0.08^{\mathrm{a}}$ & +15.79 & $8.50 \pm 0.43$ & +31.58 & $8.20 \pm 0.26$ & +26.93 \\
\hline Metformine & $5.50 \pm 0.20^{\mathrm{a}}$ & $3.71 \pm 0.08^{\mathrm{a}}$ & -67.60 & $3.42 \pm 0.06^{\mathrm{a}}$ & -62.30 & $3.64 \pm 0.06^{\mathrm{a}}$ & -66.10 \\
\hline
\end{tabular}

a differences reliable $(\mathrm{p} \leq 0.05)$ comparison to intact group of rats. 
Table 2 Types of investigated compounds' interactions with amino acid residues of the target active center according to the docking studies

\begin{tabular}{|c|c|c|}
\hline \multicolumn{3}{|r|}{ Investigated protein and types of interactions with amino acid residues } \\
\hline Comp. & $\begin{array}{l}\text { Affinity, } \\
\text { kcal/mol }\end{array}$ & KATP channel, PDB ID-6BAA \\
\hline $3 \mathbf{a}$ & -8.2 & ARG1246 ${ }^{\mathrm{a}}, \mathrm{ARG} 1300^{\mathrm{b}}, \mathrm{TYR} 377^{\mathrm{a}}, \mathrm{LEU} 434^{\mathrm{c}}, \mathrm{TYR} 377^{\mathrm{c}}, \mathrm{LEU} 52^{\mathrm{c}}$ \\
\hline $3 f$ & -8.2 & ARG1300, ASP1304 ${ }^{\mathrm{b}}, \mathrm{ILE}^{\mathrm{a}} 81^{\mathrm{c}}, \mathrm{TRP} 430^{\mathrm{c}}, \mathrm{PHE} 433^{\mathrm{c}}, \mathrm{ARG} 1300^{\mathrm{c}}, \mathrm{ALA} 1303^{\mathrm{c}}$. \\
\hline $3 \mathbf{h}$ & -8.2 & SER1238 ${ }^{\mathrm{a}}, \mathrm{ARG} 1246^{\mathrm{a}}, \mathrm{HASN} 1245^{\mathrm{a}}, \mathrm{TRP} 430^{\mathrm{c}}, \mathrm{ILE}_{381^{\mathrm{c}} \text {. }}$ \\
\hline $4 \mathbf{a}$ & -8.5 & 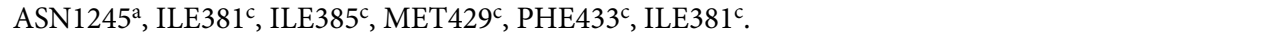 \\
\hline $4 c$ & -9.5 & 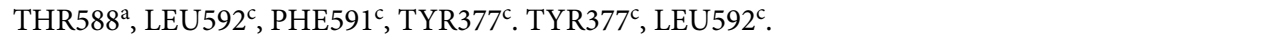 \\
\hline $4 \mathrm{f}$ & -9.5 & 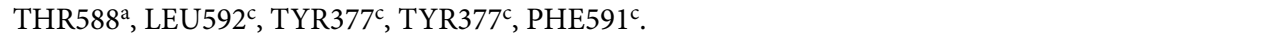 \\
\hline $4 \mathrm{~h}$ & -10.1 & 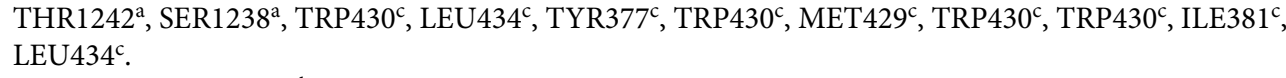 \\
\hline $4 \mathbf{i}$ & -9.0 & 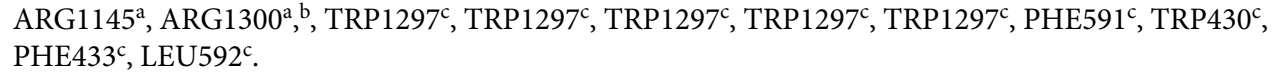 \\
\hline Mitiglinide & -7.5 & 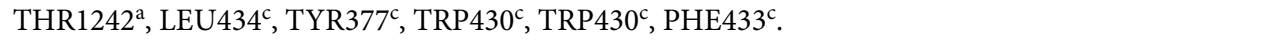 \\
\hline Glibenclamide $^{*}$ & -8.4 & 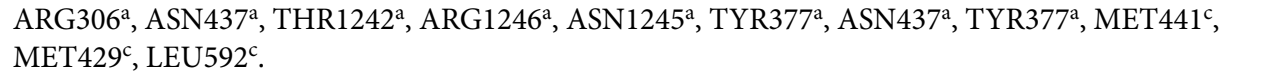 \\
\hline
\end{tabular}

${ }^{a}$ hydrogen; ${ }^{b}$ electrostatic; ${ }^{c}$ hydrophobic ${ }^{*}$ data according to X-ray structure of protein. ${ }^{32}$

4c, 4 f and 4i), taking into account the search strategy. It allowed to identify the main interaction types of the synthesized compounds, Mitiglinide and Glibenclamide with the amino acid residues with active channel centers and select compounds for further studies (Table 2). ${ }^{32}$

The visualization of the interaction of the structures with the active site of KATP channel (Figure 2) showed that compounds $\mathbf{3} \mathbf{a}$ and $\mathbf{4 a}$ revealed similar interaction to Glibenclamide. Such common interactions for compound 3a were: two hydrogen bonds with the amino acid residues

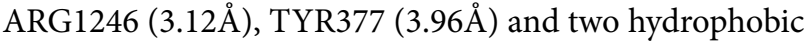

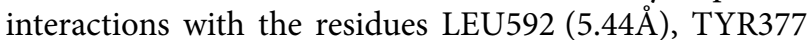

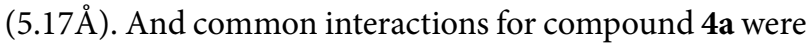
as following: hydrogen bond with the amino acid residue ASN1245 (3.76 $\AA$ ) and hydrophobic interaction with the

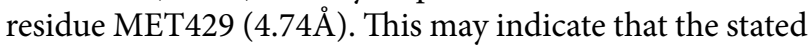
class of compounds might have ability to act in the same way as Glibenclamide does.
Hypoglycemic activity studys in the shorter of the experimental periods with a previous glucose loading allowed to establish that compounds $\mathbf{3 a}, \mathbf{3 f}, \mathbf{3 h}, \mathbf{4 a}, \mathbf{4 f}$ reduced glucose level on $19.34 \%, 17.54 \%, 18.85 \%, 25.09 \%$ and $9.51 \%$, respectively on the 15 minute of the experiment, compared to control group (Table 3). It is important that the hypoglycemic effect of these compounds is maintained throughout the experiment from 30 to 120 minutes. While compounds $\mathbf{4 c}, \mathbf{4 h}$ and $4 \mathbf{i}$ did not affect glucose level and in some cases even increased it throughout the experiment.

SAR analysis showed that the compounds with (3H-quinazoline-4-ylidene)hydrazides $(\mathbf{3 a}, \mathbf{3 f}, \mathbf{3 h})$ or $[1,2,4]$ triazolo[1,5-c] quinazoline (4a, 4f) moeities bonded through the ethyl (3a, $\mathbf{4 a})$ or phenyl (3f, $\mathbf{3 h}, \mathbf{4 f})$ linker groups with a hydrogenated isoindoline ring have hypoglycemic effect in the short term of the experiment. Modification of compounds and their further studies on hypoglycemic activity are going to be continued.

Table 3 Influence of synthesized compounds on glucose level in blood of experimental animals in the short term of the experiment

\begin{tabular}{lccccc}
\hline Comp. & $\begin{array}{c}\text { Initial } \\
\text { glucose level }\end{array}$ & $\begin{array}{c}\text { Glucose level } \\
\text { in } \mathbf{1 5} \mathbf{~ m i n}\end{array}$ & $\begin{array}{c}\text { Glucose level } \\
\text { in } \mathbf{3 0} \mathbf{~ m i n}\end{array}$ & $\begin{array}{c}\text { Glucose level } \\
\text { in } \mathbf{6 0} \text { } \mathbf{~ i n}\end{array}$ & $\begin{array}{c}\text { Glucose level } \\
\text { in } \mathbf{1 2 0} \text { min }\end{array}$ \\
\hline \multicolumn{2}{l}{ Control group $5.84 \pm 0.05$} & $6.10 \pm 0.21$ & $6.60 \pm 0.08$ & $5.62 \pm 0.14$ & $5.85 \pm 0.13$ \\
$\mathbf{3 a}$ & $4.34 \pm 0.12^{\mathrm{a}}$ & $4.92 \pm 0.59$ & $4.68 \pm 0.54$ & $4.93 \pm 1.14$ & $3.84 \pm 0.51$ \\
$\mathbf{3 f}$ & $5.12 \pm 0.15^{\mathrm{a}}$ & $5.03 \pm 0.14^{\mathrm{a}}$ & $4.87 \pm 0.19^{\mathrm{a}}$ & $5.23 \pm 0.12^{\mathrm{a}}$ & $5.12 \pm 0.34$ \\
$\mathbf{3 h}$ & $4.48 \pm 0.23$ & $4.95 \pm 0.21$ & $4.92 \pm 0.19^{\mathrm{a}}$ & $5.41 \pm 0.24$ & $5.32 \pm 0.16^{\mathrm{a}}$ \\
$\mathbf{4 a}$ & $3.98 \pm 0.13^{\mathrm{a}}$ & $4.63 \pm 0.31$ & $4.52 \pm 0.22$ & $4.49 \pm 0.14^{\mathrm{a}}$ & $5.54 \pm 0.11^{\mathrm{a}}$ \\
$\mathbf{4 c}$ & $5.83 \pm 0.27$ & $6.23 \pm 0.17^{\mathrm{a}}$ & $6.93 \pm 0.26$ & $6.08 \pm 0.19^{\mathrm{a}}$ & $6.12 \pm 0.20$ \\
$\mathbf{4 f}$ & $5.34 \pm 0.22$ & $5.52 \pm 0.23$ & $5.48 \pm 0.22$ & $5.52 \pm 0.15^{\mathrm{a}}$ & $5.32 \pm 0.17^{\mathrm{a}}$ \\
$\mathbf{4 h}$ & $5.87 \pm 0.25$ & $6.13 \pm 0.31$ & $6.54 \pm 0.27$ & $5.89 \pm 0.24$ & $5.93 \pm 0.21$ \\
$\mathbf{4 i}$ & $6.10 \pm 0.21$ & $8.50 \pm 0.58$ & $6.78 \pm 0.85$ & $6.34 \pm 0.49$ & $5.98 \pm 0.38$ \\
\hline
\end{tabular}

a differences reliable $(\mathrm{p} \leq 0.05)$ comparison to control group of rats. 
(a)

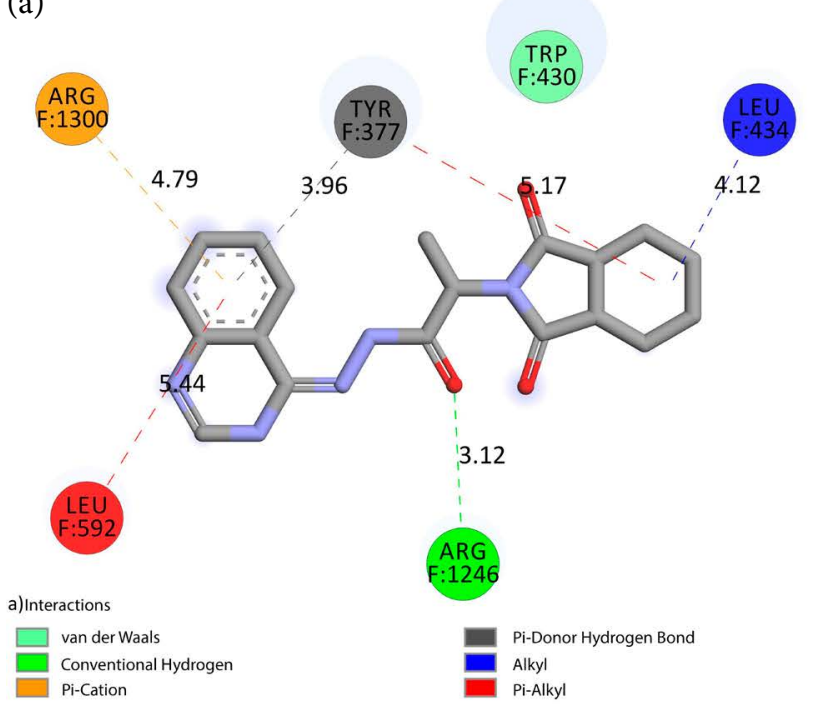

(c)
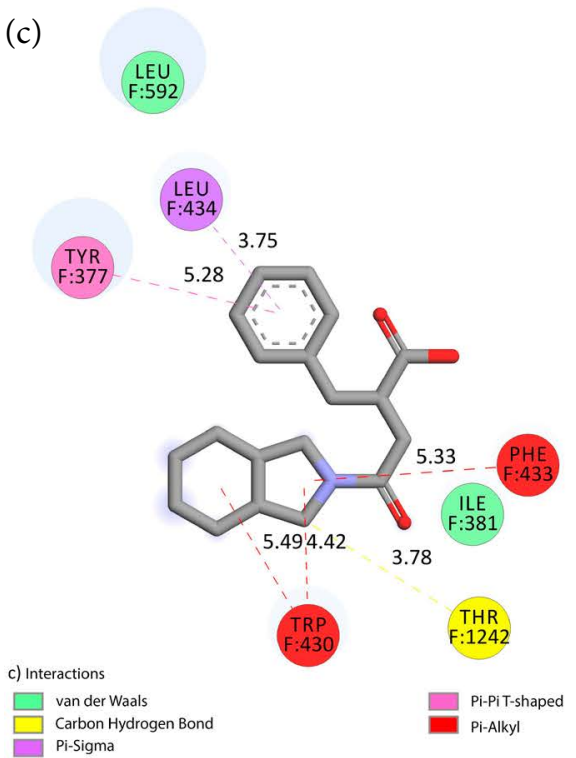

(b)

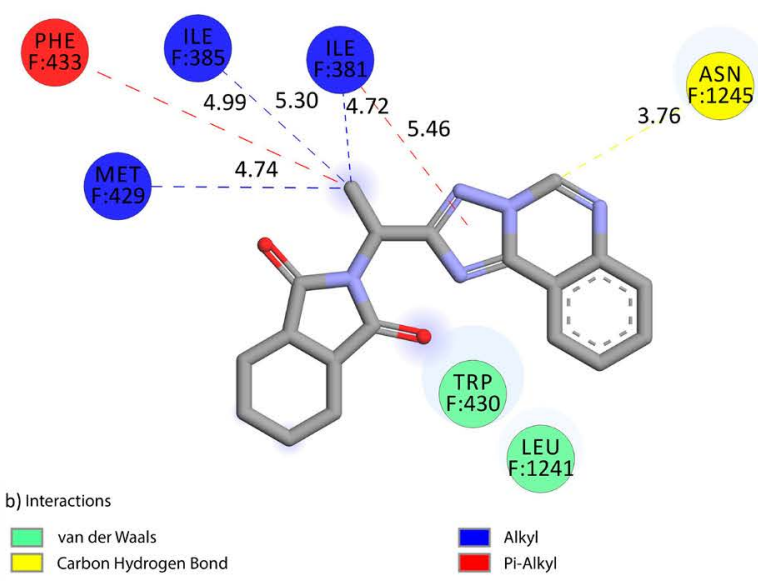

(d)

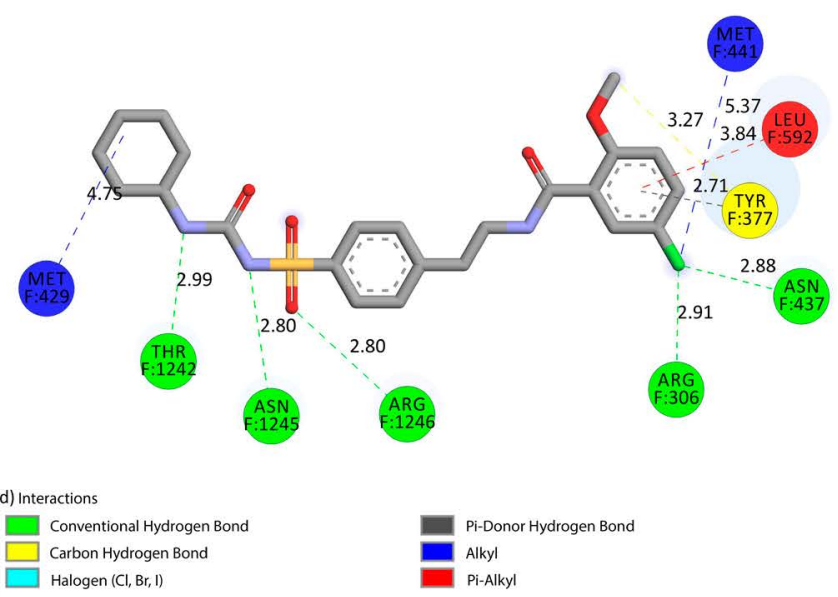

Figure 2. Visualization of affinity according to the docking (a) compound 3a with KATP channel; (b) compound 4a with KATP channel; (c) Mitiglinide with KATP channel; (d) Glibenclamide with KATP channel.

\section{Conclusions}

The synthesis of $\mathrm{N}$-carboxyalkyl-(arylalkyl-,aryl-)-isoindoline-1,3-dione (3H-quinazoline-4-ylidene) hydrazides was carried out using activated $\mathrm{N}$-protected aminoacids and 4-hydrazinoquinazoline. The synthesized hydrazides were converted into the corresponding hydrogenated $2-([1,2,4]$ triazolo $[1,5-c]$ quinazoline-2-yl)-alkyl-(alkylaryl-,aryl-)isoindole-1,3(2H)-diones by cyclocondensation under the conditions of acidic catalysis. The structures of the synthesized compounds were established using IR, ${ }^{1} \mathrm{H}$ and ${ }^{13} \mathrm{C}$ NMR spectroscopy and LC-MS and the features of spectral pattern were discussed. Search strategy of hypoglycemic drugs based on the fragment-oriented design revealed a number of perspective compounds, which are short-acting drugs, like prandial glucose regulators. Conducted SAR analysis showed that the introduction of hydrogenated 1,3-dioxoisoindole moiety bonded via a linker group with 4-hydrazynoquinazoline and triazolo[1,5-c] quinazoline cycle is reasonable in the context of searching for short-acting hypoglycemic agents and requires further research.

\section{Acknowledgments}

The work was performed with the financial support of «Enamine Ltd» (Kyiv, Ukraine). 


\section{References}

1. B. M. Santosh, P. A. Narshinha, Tetrahedron 2006, 62, $9787-$ 9826. DOI:10.1016/j.tet.2006.07.098

2. J. P. Michael, Nat. Prod. Rep. 2007, 24, 223-246. DOI:10.1039/b509528j

3. U. A. Kshirsagar, Org. Biomol. Chem. 2015, 13(36), 93369352. DOI:10.1039/C5OB01379H

4. A. N. Parvez, R. Arpana, M. Imran, Int. J. Pharm. Biol. Arch. 2011, 2(6), 1651-1657.

5. P. S. Theivendren, V. K. Palanirajan, Res. In Pharm. 2011, 1(1), $1-21$.

6. G. Marzaro, A. Guiotto, A. Chilin, Expert Opin. Ther. Pat. 2012, 22(3), 223-252. DOI:10.1517/13543776.2012.665876

7. S. Ravez, O. Castillo-Aguilera, P. Depreux, L. Goossens Expert Opin. Ther. Pat. 2015, 25(7), 1-16.

DOI:10.1517/13543776.2015.1039512

8. A. O. Olayinka, Y. A. Oluwatosin, D. V. Aderohunmu, F. E. Owolabi, A. O. Olomieja. Am. J. Drug Discov. Dev. 2017, 7, 1-24. DOI:10.3923/ajdd.2017.1.24

9. S. Srivastava, S. Srivastava, Int. J. Pharm. Sci. Res. 2015, 6(9), 1206-1213. http://www.ijpsr.info/docs/IJPSR15-06-09-102.pdf.

10. D. Wang, F. Gao, Chem. Cent. J. 2013, 7(95), 1-15. DOI:10.1186/1752-153X-7-95

11. I. Khan, A. Ibrar, N. Abbas, A. Saeed, Eur. J. Med. Chem. 2014, 76, 193-244. DOI:10.1016/j.ejmech.2014.02.005

12. A. Mohammad, Int. J. Med. Chem. 2014, 27, 1-27. DOI:10.1155/2014/395637

13. V. G. Ugale, S. B. Bari, Eur. J. Med. Chem. 2014, 80, 447-501. DOI:10.1016/j.ejmech.2014.04.072

14. O. Voskoboynik, S. Kolomoetsch, G. Berest, I. Nosulenko, Yu. Martunenko, S. Kovalenko, Chem. Heterocycl. Compd. 2017, 53(3), 256-272. DOI:10.1007/s10593-017-2048-2

15. A. G. Reddy, V. H. Babu, Y. J. Prakash Rao, JCPS 2017, 10(3), 1492-1504

16. Z. Chaban, St. Harkov, T. Chaban, O. Klenina, V. Ogurtsov, I. Chaban, Pharmacia 2017, 64(3), 52-66. https://www.researchgate.net/publication/322731545.

17. A. Hameed, M. al-Rashida, M. Uroos, S. A. Arshia, M. Ishtiaq, K. M. Khan, Expert Opin. Ther. Pat. 2018, 28(4), 281-297. DOI:10.1080/13543776.2018.1432596
18. O. Antypenko, S. Kholodnyak, K. Schabelnyk, L. Antypenko, S. Kovalenko, Chem. Heterocycl. Compd. 2017, 53(3), 292309. DOI:10.1007/s10593-017-2051-7

19. O. Kolomoets, O. Voskoboynik, Ol. Antypenko, G. Berest, I. Nosulenko, V. Palchikov, O. Karpenko, S. Kovalenko, Acta Chim. Slov. 2017, 64, 902-910. DOI: 1https://doi.org/10.17344/acsi.2017.3575

20. P. J. Hajduk, J. Greer, Nat. Rev. Drug Discov. 2007, 6(3), 211219. DOI: $10.1038 / \mathrm{nrd} 2220$

21. D. A. Erlanson: Introduction to Fragment-Based Drug Discovery, In: Fragment-Based Drug Discovery and X-Ray Crystallography, T. G. Davies, M. Hyvönen Eds., Berlin Heidelberg: Springer Verlag, 2012, 1-32. DOI:10.1007/128_2011_180

22. Yu. Martunenko, M. Kazunin, O. Antypenko, Ye. Selivanova, S. Kovalenko, S. Trzhetsynskyi, Current issues in pharmacy and medicine: science and practice, 2018, 11(1), 4-11. DOI: $10.14739 / 2409-2932$

23. H. Ohnota, T. Koizumi, N. Tsutsumi, M. Kobayashi, S. Inoue, F. Sato, J. Pharmacol. Exp. Ther. 1994, 269(3), 489-495.

24. W. J. Malaisse, Expert. Opin. Pharm. 2008, 9(15), 2691-2698. DOI:10.1517/14656566.9.15.2691

25. https://www.drugbank.ca/unearth/q?c=_score\&d=down\& query=quinazolines\&searcher=drugs.

26. Protein Data Bank, pdb. Retrieved from http://www.pdb.org.

27. MarvinSketch version: 17.21.0, ChemAxon (http://www.chemaxon.com)

28. O. Trott, A. J. Olson, J. Comput. Chem. 2010, 31, 455-461. DOI:10.1002/jcc.21334

29. S. Kovalenko, L. Antypenko, A. Bilyi, S. Kholodnyak, O. Karpenko, O. Antypenko, N. Mykhaylova, T. Los', O. Kolomoets', Sci. Pharm. 2013, 81(2), 359-392.

DOI:10.3797/scipharm.1211-08

30. El H. Sayed, El Ashrya, S. Nadeem, M. R. Shah, Ye. El Kilanyd, Adv. Heterocycl. Chem. 2010, 101, 161-228.

31. E. Breitmaier, Structure Elucidation By NMR In Organic Chemistry: A Practical Guide, John Wiley \& Sons, Ltd. 2002, 258. DOI:10.1002/0470853069

32. M. M. Gregory, B. Kandasamy, F. DiMaio, C. Yoshioka, S. Show-Ling, eLife 2017, 6. DOI:10.7554/eLife.31054

\section{Povzetek}

Opisujemo učinkovito sintezo (3H-kinazolin-4-iliden)hidrazidov $N$-karboksialkil-(arilalkil-,aril-)izoindolin-1,3-dionov iz aktiviranih $N$-zaščitenih amino kislin in 4-hidrazinokinazolinov. Predlagana metoda temelji na neposrednem iskanju hipoglikemičnih agentov s pomočjo načrtovanja, orientiranega na fragmentih, in z molekulskim sidranjem. Željene hidrazide smo pripravili s pomočjo ciklokondenzacij pod kislimi pogoji in jih nadalje pretvorili v ustrezne 2-([1,2,4] triazolo[1,5-c]kinazolin-2-il-)alkil-(alkilaril-,aril-)-hidroizoindol-1,3(2H)-dione. Strukture pripravljenih spojin smo določili s pomočjo IR, ${ }^{1} \mathrm{H}$ in ${ }^{13} \mathrm{C}$ NMR spektroskopije ter LC-MS analize ter s primerjavo spektralnih vzorcev (kar je opisano v diskusiji). Rezultati farmakološkega testiranja so razkrili serijo spojin, ki delujejo kot hitri hipoglikemični agenti, podobno kot prandial regulira glukozo (Mitiglinid). SAR analiza je dodatno pokazala, da je vpeljava hidrogeniranega 1,3-dioksoindolnega fragmenta, ki je preko distančnikov vezan na 4-hidrazinokinazolinski ter triazolo[1,5-c]kinazolinski obroč, primerna za iskanje novih hitro delujočih hipoglikemičnih agentov ter da si zasluži nadaljnje raziskave. 